\title{
Early metabolic differences between obesity-resistant and obesity-prone mice: role of adipokines
}

\author{
Petra Janovska $^{1}$, Kristina Bardova ${ }^{1}$, Olga Horakova ${ }^{1}$, Jana Hansikova ${ }^{1}$, Vladimir Kus ${ }^{1}$, \\ Evert M. van Schothorst ${ }^{2}$, Femke P.M. Hoevenaars ${ }^{2}$, Melissa Uil ${ }^{2}$, Michal Hensler ${ }^{1}$, \\ Jaap Keijer ${ }^{2}$ and Jan Kopecky ${ }^{1}$ \\ ${ }^{1}$ Department of Adipose Tissue Biology, Institute of Physiology of the Czech Academy of Sciences, Videnska 1083, 142 \\ 20 Prague, Czech Republic and ${ }^{2}$ Human and Animal Physiology, Wageningen University, De Elst 1, 6708 WD \\ Wageningen, The Netherlands
}

Both genetic and environmental factors contribute to development of obesity and its adverse metabolic consequences. Multiple regulatory pathways are involved including their circadian rhythms. Thus, adipokines, bioactive peptides and proteins secreted by adipose tissue, may play an important role in energy homeostasis and in the development of metabolic syndrome. In mice, leptin exhibits rhythmic production across the light-dark cycle and in obese humans, disruption of the 24 hour profiles of leptin and adiponectin was observed in comparison to healthy lean subjects (Maury et al. 2010). Novel experimental models are required to better characterize the complex involvement of circadian rhythms and adipokines in regulation of energy metabolism.

The goal of this study were (i) to learn whether obesity-resistant A/J and obesity-prone C57BL/6J (B6) mice differ in their metabolic flexibility (i.e. their potential to adjust fuel oxidation to fuels availability) right after weaning; (ii) to compare glucose tolerance tests (GTT) with indirect calorimetry (INCA) in the assessment of metabolic flexibility to glucose in the post-weaning mice; and (iii) to assess the role of adipokines, leptin and adiponectin - including the daily oscillations in their plasma levels, in the metabolic response in two mice strains with different genetic background. $\mathrm{A} / \mathrm{J}$ and $\mathrm{B} 6$ male mice were maintained at $20{ }^{\circ} \mathrm{C}$ and weaned to standard lowfat diet at 30 days of age. During the first day after weaning (i) GTT (either oral, OGTT; or intraperitoneal, IPGTT) at $20^{\circ} \mathrm{C}$, and (ii) INCA, during a fasting/re-feeding transition (with respecting the circadian rhythm of feeding in mice), were performed. INCA was conducted in fasted and re-fed state, at thermoneutral temperature $\left(34^{\circ} \mathrm{C}\right)$, thus avoiding the confounding effect of stimulation of non-shivering thermogenesis. Levels of plasma adipokines in fasted and re-fed state were measured in the post-weaning mice, and the circadian rhythms in their levels were characterized in adult animals.

Results indicate higher glucose tolerance (i.e. lower incremental AUC of glucose both IPGTT and OGTT) and higher metabolic flexibility in $\mathrm{A} / \mathrm{J}$ mice as compared with B6 mice. In fasted state, respiratory quotient (RQ) was not affected by the strain. In response to re-feeding, RQ increased in both strains $(p<0 \cdot 01)$, with faster response in A/J as compared with B6. When evaluated between 0:00 a.m. and 2:00 a.m. (6-8 hours after re-feeding), RQ values were higher in A/J mice than in B6 mice (A/J vs. B6; $1.00 \pm 0.03$ vs. 0.92 \pm 0.02$)$. Leptin levels were affected by nutrition state, its plasma levels increased in re-fed state in comparison to fasted state (A/J $\sim 2.3$ fold and B6 3.4 fold) and the levels of leptin were higher in A/J mice as compared with B6 (A/J vs. B6; in fasted state mice $\sim 3.9$ fold increased and in re-fed state $\sim 2.6$ fold increased). The nutritional state had no impact on adiponectin levels in plasma and the levels of adiponectin were higher in B6 as compared with A/J. Different regulation of plasma leptin during 24 hours was found in adult mice fed low (ST) as compared high fat (HF) diet, with higher a oscillation in $\mathrm{A} / \mathrm{J}$ mice; in both strains, the levels culminated around 8.00 p.m. (Fig. 1).

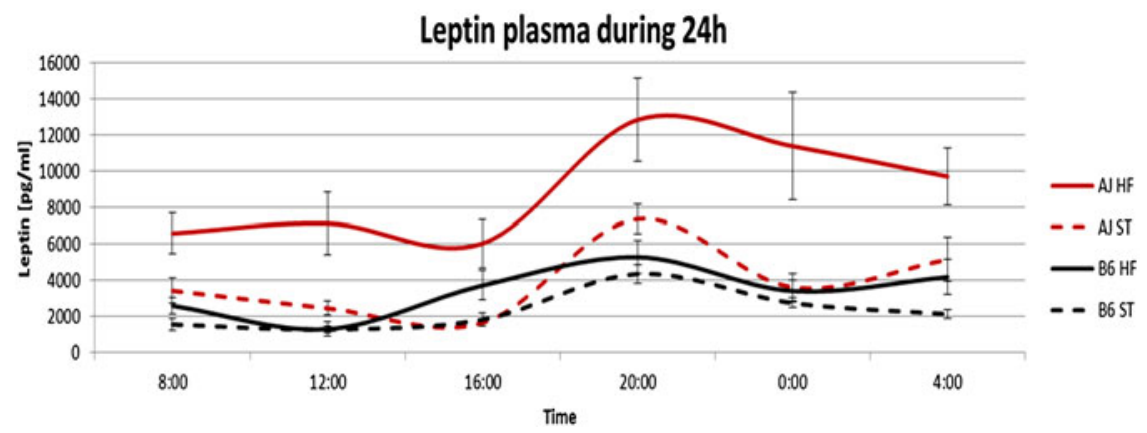

Fig. 1 Plasma leptin levels during 24 hours measured in adult mice using Bio-Plex system. Data are means \pm SE $(n=6-8)$.

Conclusions: These differences in control of glucose metabolism and levels of adipokines, whose oscillation may be important for their impact on metabolism, could be linked to the different genetically-determined propensity to obesity of the mice.

This work was supported by the grants from the Ministry of Health of the Czech Republic (NT14250-3/2013 and NT13763-4).

Maury E, Ramsey KM \& Bass J (2010) Circ Res 106, 447-462. 